\title{
Scheduling Railway Lines
}

\author{
Tanuj Dey, Rahul Bal, B.Bharathi
}

\begin{abstract}
This paper covers the primary purpose of reserving and undertaking plan of unidirectional and bi-directional via an software that covers the administrations of evaluation the educate calendar as steady with the client's login, alongside an administrator login which includes the precept purpose of along with/converting the educate facts as in step with the changes in the timings of the teach plan. The software moreover makes the paintings easy for the teach dispatcher, because the execution of the teach dispatcher is made absolutely mechanized thru this method. One of the greater issue gave in that is, the consumer file made with an enrolled portable huge variety can be actuated for SMS cautions for any enlisted teach, which makes it all through the board software. The precept dreams to be managed on this paper is that (I) it's miles an throughout the board software, which spreads customer and administrator desires beneath one (ii) It makes crafted thru educate dispatcher "really automatic" (iii) It offers SMS ready control under the enrolled file for the educate booked below it.
\end{abstract}

\section{INTRODUCTION}

Usage on Railway and Railway Scheduling has been chipped away at for pretty a long term and years now. a couple of views had been worked and actualized effectively in couple of nations. Multiple viewpoints have continuously been hypothetically secured but never actualized on actual due to the unsure consequences and finishes of these strategies. Multiple global locations fall behind in Railway Scheduling because of the majority of the facts and information being administrative art work position in place of a automatic company. The complete notion of Reinforcement getting to know is for the device to have the choice to look at without everybody else after an important association of powerful informational series is reinforced into the machines. To have all the gadget walking in an effective way it is top notch that the statistics in a automatic association.

Usually a educate dispatcher is given records thru the railroad stations manage workplace and after that the important increase is taken through the teach dispatcher to redirect into the particular heading. This precise way has been finished into a totally programmed technique in couple of nations, however applying it in India may be in the suggest time without a doubt be finished with the aid of illuminating the dispatcher through an utility. A mechanized SMS is one of the maximum required highlights in any motion/statistics

application as it spares a consumer's time. A huge amount of

Revised Version Manuscript Received on July 10, 2019

Tanuj Dey, Student, Department of Computer Science and Engineering, Sathyabama Institute of Science and Technology, Chennai-119, Tamil Nadu, India.(Email: tanuj200@gmail.com)

Rahul Bal, Student, Department of Computer Science and Engineering, India.(Email: rahulbal0601@gmail.com).

B.Bharathi, Professor, Department of Computer Science and Engineering Sathyabama Institute of Science and Technology, Chennai-119, Tamil Nadu, India.(Email: bharathivaradhu@gmail.com). Sathyabama Institute of Science and Technology, Chennai-119, Tamil Nadu,

the person adventure applications supply a SMS organized framework, but an software program to hold all under one rooftop is the element that we're looking in advance here.

\section{A. Problem Declaration}

The precept purpose is to make an software that could cover the inquiries of the consumer approximately train Scheduling and Platform Scheduling. Likewise to offer an administrator login in which the educate subtleties can be altered and adjusted through necessities and after that which offers the information to the dispatcher. A SMS organized framework likewise made to deliver every one of the highlights below one window.

\section{B. Hassle Description}

A massive trouble is with characterizing exceptional belongings at the railroad strains, with song numbers, degree numbers and the empty song as indicated by the teach spaces. the principle hassle to be managed is the planning of trains as in step with source, intention and time of touchdown, if you want to inevitably be primary in managing the time timetable exhibit inside the transportable application. even as that is handled, the timings and rerouting of the trains grow to be less difficult for the dispatcher. when the educate is dispatched into its separate bearing, at that factor the vacationers may be knowledgeable about the diploma quantity and timing of the educate in my view.

\section{LITERATURE REVIEW}

Valentina Cacchiani, Alberto Caprara, and Paolo Toth [1] in their paper approximately "Heurestic method for train Unit project" learn about a streamlining problem on railroads known as the train Unit task hassle. A teach unit consists of a self reliant train with an engine and a ton of wagons with voyager seats. Given a outstanding deal of timetabled teach journeys, each with a required wide variety of voyager seats, and a ton of train devices, every with a given quantity of available seats, the difficulty requires the nice errand of the train gadgets to the tours, conceivably merging a couple of train unit for a given trek that fulfills the seat requests.

A. Higgins, E. Kozan and L. Ferreira within the paper "Optical Scheduling of Trains on a unmarried Line music" [2] have located about a railroad streamlining hassle called the educate Unit venture hassle. A train unit incorporates of an unbiased train with a motor and a number of wagons with visitor seats. Given an expansion of timetabled train journeys, every with a required number of traveler seats, and plenty of educate gadgets, each with a given number of handy 
seats, the issue requires the great mission of the teach devices to the outings, possibly consolidating a couple of educate unit for a given time out that satisfies the seat needs.

In the paper "consistent teach reserving: from speculation to rehearse" [3] paintings expounds at the essential results of a continual task furnished for evaluate the sensible pertinence of dispersed strategies for all the manner right down to business company progressing educate arranging. This paper covers all the lucidly proposed mind inside the theoretical appearing to making use of it dynamically outcomes. The last purpose of the venture is the improvement of an impelled choice honestly sturdy tool for assisting dispatchers paintings and for guiding them toward close to perfect everyday train re-timing, re-citing and re-directing decisions.

The makers "Wei Fang, Shengxiang Yang, Xin Yao"[4] in their paper in 2015 gift an exhaustive survey on brilliant problem fashions for rescheduling in railroad organizes thru an obvious collecting. a few every so often used fashions are depicted in detail through searching over their factors and desires. This paper moreover bases at the course of motion philosophies proposed in the composition.

The paper "educate guiding principle in single-track journey frameworks" [5] composed with the aid of "Marco Galaverna, Alberto Imovilli and Giuseppe Sciutto" rehashes the preliminary phase of an examination which is going for evaluating the presentation of single-track travel frameworks and enhancing it via methods for appropriate guiding principle structures of train jogging. right off the bat, hypothetical execution is assessed with the aid of accepting conventional undisturbed interest; this research goes for giving an arranging stage approach to affirm whether or no longer single following is cheap for a specific software.

\section{METHODOLOGIES \& RESULTS}

The primary target of the calculation is to get the railroad traces planned to artwork in a way, it really is best to the heuristic technique. The heuristic calculation should be expert to address severa models of control event and educate structures, so it thoroughly may be overseen in a sensible manner but heaps as might also need to fairly be predicted and function the choice to supervise and paintings as a minimum trains moving at an identical heading and manner. The primary necessity is to present a manual of explicit state to a summed up kingdom area of constant length. At that point the decentralization for character trains is finished with limiting the vector to a set skyline. At that aspect the request for train's development is gotten the usage of an occasion trigger the use of the halt shirking of heuristic set of regulations.[5]

The most massive three difficulties that must be routed to perform the above goals are: Ii wonderful framework administrations and diverse educate management activities have to be handled productively through the calculation, ii)the version proposed should have the potential to scale to realistic railroad tracks, iii) the calculation have to cope with activities of synchronous shifting trains.

A.Generalized United States of America illustration For each desire for any character train want to be taken, an individual us of a vector is drawn and processed. The belongings are checked from the heading of development of train in frontwards and in opposite positions. $\mathrm{Lb}$ and $\mathrm{Lf}$ (referred to as neighborhood property) are applied to talk to person state vectors in opposite and beforehand route one after the opposite. Multiple assets are managed to pave way for the fast moving trains ahead. In the below figure, $\mathrm{Lb}=2$ and $\mathrm{Lf}=6$.

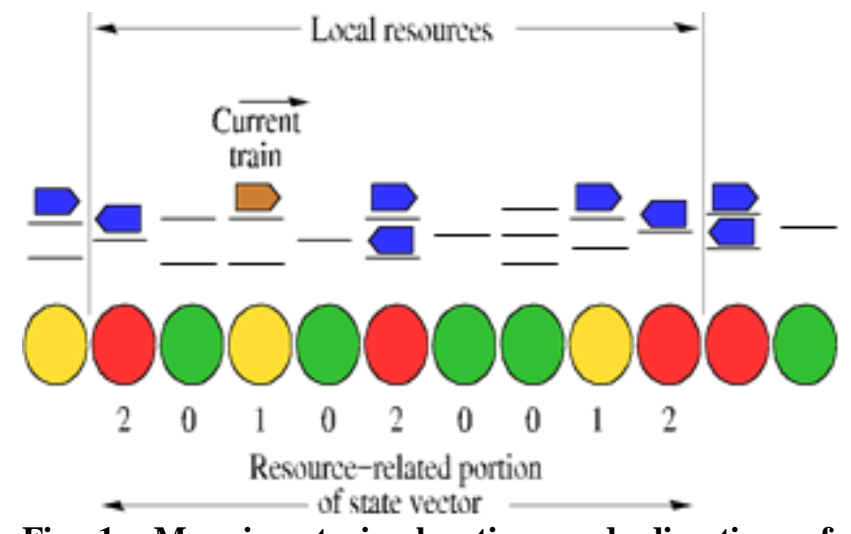

Fig 1: Mapping train location and direction of movement to resource status, relative to the 'current train'.

As shown in Figure 1, let us assume each state vector $(\mathrm{Sr})$ resources takes the values from $1,2 \ldots \mathrm{R}-1$ resources $(\mathrm{R})$. Let's consider $\mathrm{R}=3$, as higher values gives us higher congestion within the resources. If we consider $\mathrm{R}=3$ we get $\mathrm{Sr}$

$=\{0,1,2\}$. Let's consider $\mathrm{Nr}$ to be total number of tracks in which Trc is trains moving towards and Trd is diverting away from current train. Since only one train can be accommodated per track Trc $+\operatorname{Trd}<=\mathrm{Nr}$.

$$
\mathrm{Sr}=\mathrm{R}-1-\min \left(\mathrm{R}-1,\left(\mathrm{Nr}-\mathrm{W}_{\mathrm{c}} \mathrm{Trc}-\mathrm{W}_{\mathrm{d}} \mathrm{Trd}\right)\right)
$$$$
\rightarrow(1)
$$

Wc and Wd have an incentive among 0 to as a minimum one which recognizes the upcoming and veering trains. at the off chance that we set $\mathrm{Wc}$ and $\mathrm{Wd}=1$ and given that $\mathrm{R}=3$, we get at any rate tracks as empty if $\mathrm{Sr}=$ zero and 1 within the event that one tune is empty and more than one if $\mathrm{R}$ is non-empty. The progressions in $\mathrm{Wd}$ and $\mathrm{Wc}$ movements the trains inverse way less complicated than the trains comparative way.

The need of modern train is likewise taken into consideration as static. The vector of period $(\mathrm{Lb}+\mathrm{Lf}+2)$ together with variety need esteem and ahead and in contrary assets +1 is taken into consideration sections of network assets. This properly worth is unaffected with the useful resource of quantity of trains or their headings. It remains equal for any better duration difficulty.

\section{A. Action and Policy Definition}

Each state vector is mapped, which takes an action which generally is binary. 0 and 1 are the states in which train takes a decision for moving ahead and halt respectively for a defined time. The decision making is repeated again and again $t$ ill the train is in halt state. 


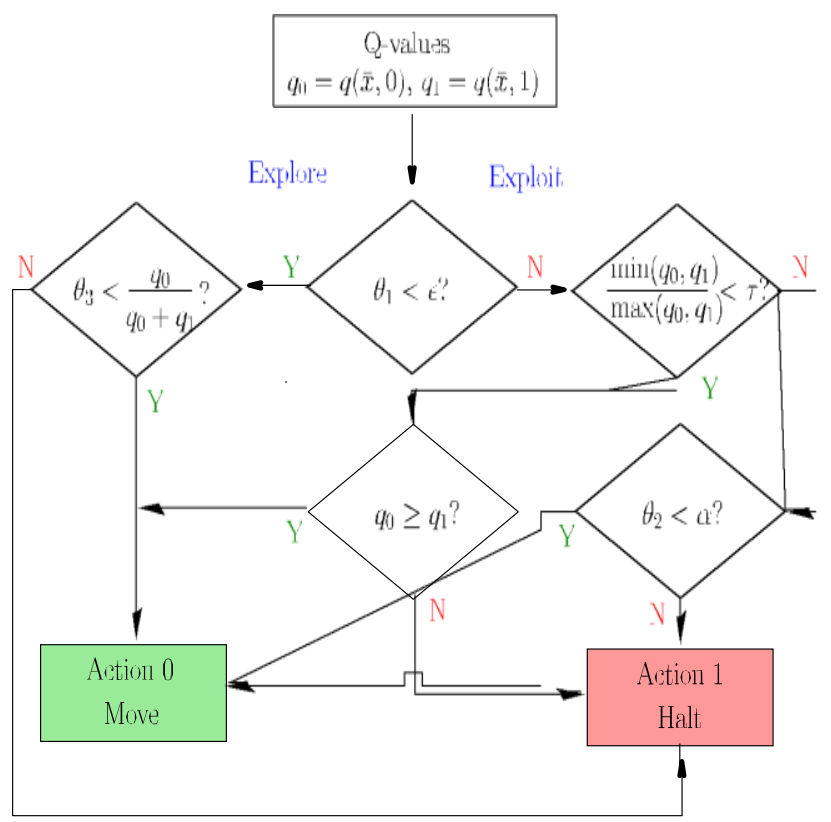

Fig 2: Flowchart of choosing actions where $\theta i$ is in range $[0,1]$.

As the above diagram (Fig 2) suggests, the greedy approach policy is used in the algorithm for the purpose of action selection. There are 2 possible actions, a $€\{0$ : move, 1: halt $\}$ which are unique from each other. Each state action pair is connected with a

Q- value $(x, a) \longrightarrow(q, a) \rightarrow(2)$

The higher teQ value the higher the desirability the greedy policy chooses the greedy option with probability $(1-€)$. There are two modes to this (i) Exploration mode: In which the decision making is like a toss with a biased coin. (ii) Exploit mode: a further biased coin is used to take the decisions, q0 q1 within a threshold $\mathrm{T}$. The bias is a user defined parameter $(\alpha)$, which is the major decision maker in choosing [6].

\section{B. Objective Function and $Q$-values}

A few other objectives to be considered in these are reducing the delay time as much as possible to the convenience of the passenger using priority weighted delay [7]. The average of this delay is calculated by mean of all trains positioned over all stations.

$$
\mathrm{J}=1 / \mathrm{N} \mathrm{r,t} * \sum \delta \mathrm{r}, \mathrm{t} / \mathrm{Pt} \longrightarrow(2)
$$

where $\delta \mathrm{r}, \mathrm{t}$ is the delay for the train $\mathrm{t}$, on departure from resource $r,[9] \mathrm{Pt}$ is the priority of the train $\mathrm{t}$ and $\mathrm{Nr}, \mathrm{t}$ is given as the total number of departures given in the schedule. The above formula is used to evaluate the minimal value of J. One of the disadvantages to be overcome is that, $\mathrm{J}$ represents the sum of overall delay. hence the put off of one train prompts errand of different trains, and also the expense of dispose of 1 train is variable to the elective trains. In the event that we measure prizes to delays, it could make inconveniences while changing over from one to some other model. also, turn around checking is beyond the realm of imagination because of reality scene is excessively delayed. rather, keeping up objective programming as a main priority, an acclaim machine is utilized. The greatest acknowledgment level for $\mathbf{J}$ is prepared at $(1+\rho)$ in which the least of $J$, for the intention that $\rho>0$. An applause for +1 and -1 is given with perceive to accomplishment (inside limit) and disappointment (impasse situation).

Fig 1: Mapping train location and direction of movement to resource status, relative to the 'current train'. The $Q$ expense characterizes the country movement sets, which is presumably spoken to as probability of accomplishment in nation development pair. higher than going by means of the entire accumulation, a parallel charge (b) genuine is set each time situated in a given circumstance. in some undefined time later on for conclusive touch or suspend condition, E.x,a is the amount of all circumstances that gave through ( $\mathrm{Ex}$, an) in any event as fast as, and E*.X,a is the gigantic assortment of those that completed with progress. $\sigma(x, a)$ speaks to protracted cutting edge situations. commendation components are created after of fulfillment and there might be concurrent training of country movement sets.

Initially, E*.x,a measures terminal acclaim. second, a one-advance once more spread of $\sigma(\mathrm{x} 1$, an) is performed while the scene is walking. The memory keeps the most contemporary state-activity pair $\sigma$ (x1, a) through each educate, and the accompanying nation movement pair situated through the equivalent instruct is set apart as a 'neighbor' (x1, a1).[9] each pair (x1, a) will have various (state $\mathrm{M}$ ) buddies (x1, a1) for the span of a scene. The normal achievement charge $\sigma(. \mathrm{X} 1, \mathrm{a} 1)$ of all $\mathrm{M}$ buddies administrative work a piece of the Q-Value.[9]

$\mathrm{q}(\mathrm{x} 1, \mathrm{a})=\mathrm{w} \sigma(\mathrm{x} 1, \mathrm{a})+(1-\mathrm{w}) \sum(\mathrm{m}=1$ to $\mathrm{M}) \sigma(\mathrm{x} 1 \mathrm{~m}$,

$\mathrm{a} 1 \mathrm{~m}) / \mathrm{M} \rightarrow(3)$

Where $\mathrm{w}$ gives the weighting factor of any given pair between the success rate, and the average success rate of its $\mathrm{M}$ neighbors. Since these are not unique neighbors are more frequently observed. Updating becomes quite difficult as since only current and average values of $\mathrm{M}$ are required.

\section{Description of Integrated Algorithm}

As the below figure (Fig 3) suggests, the simulator is run on various episodes with each starting with basic value of train. A list of events is created irrespective of a train is stationary or moving, and each event observes the time and resources taken and the last completed action along with its direction of movement.

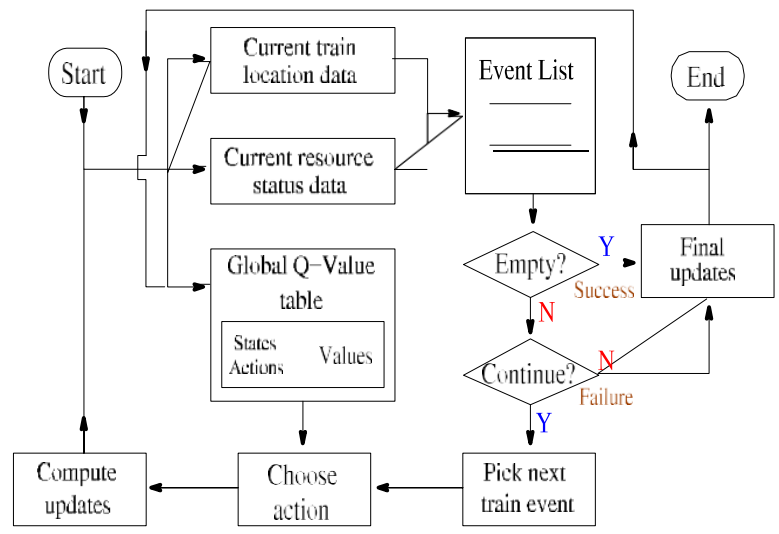

Fig 3. Scheduling algorithm flowchart 
at the off hazard that a educate is desk sure, the occasion handling time is the most punctual flight time possible for the educate, and invest negligible energy and through aany requirements on the takeoff time. If there ought to stand up an incidence of similar cause, it is additionally determined utilizing least separation voyaged and the speed. In each development, the maximum decreased time stamp in rundown is discovered. At a given time stamp if there may be occasion of numerous activities, they're saved going for walks in a consecutive request via save you shirking calculation as recognized in the writing. The grouping of occasions is orchestrated with the give up reason that maximum muddled assets of the trains are applied first.

The decrease the quantity of loose tracks in an asset, the better the entanglement, and the sooner the train possesses that asset. at the off danger that an event is treated as soon as, the nation vector is worked for the educate. An pastime is selected the use of the country vector, the specific Q-values, and the atypical technique for selecting if or now not to differentiate and use the mastering received up until this factor. The interest is connected thru the reproduction motor, which refreshes (I) beginning location and asset, (ii) refreshed vicinity and belongings (iii) the Q-esteem desk. The undertaking motor ensures that each one alternatives are feasible. Infeasible choices are given a punishment thru resetting the success of the u . s . a .-interest pair to zero, and turning around it. only a transferring may be invalid when you consider that give up is the fundamental situation of train. The calculation rehashes iterative until all trains arrive at their given desires efficiently or atleast till the fulfillment of a sadness condition.

\section{D.SMS Alert gadget}

In each single composed framework, in which era the executives is a noteworthy problem the framework is worked with a programmed cautioning framework thru content informing. Time the board assumes significant challenge within the accomplishment of any undertaking. it's far vital to help or take away darkness from the overall populace to take into account their separate time plan to cause them to attain in advance than required for his or her arrangements. hand-held gadgets, for example, cell telephones serve the most huge project of contacting humans correctly impartial in their vicinity. consequently, there's the need to build up a framework which will join accessible belongings and get in touch with individuals via short message management (SMS) the use of GSM module. The framework engineering is appeared in decide four and it applied using SMS over circuit exchanged GSM prepare that allows the software to ship and get messages over the modem interface [8].

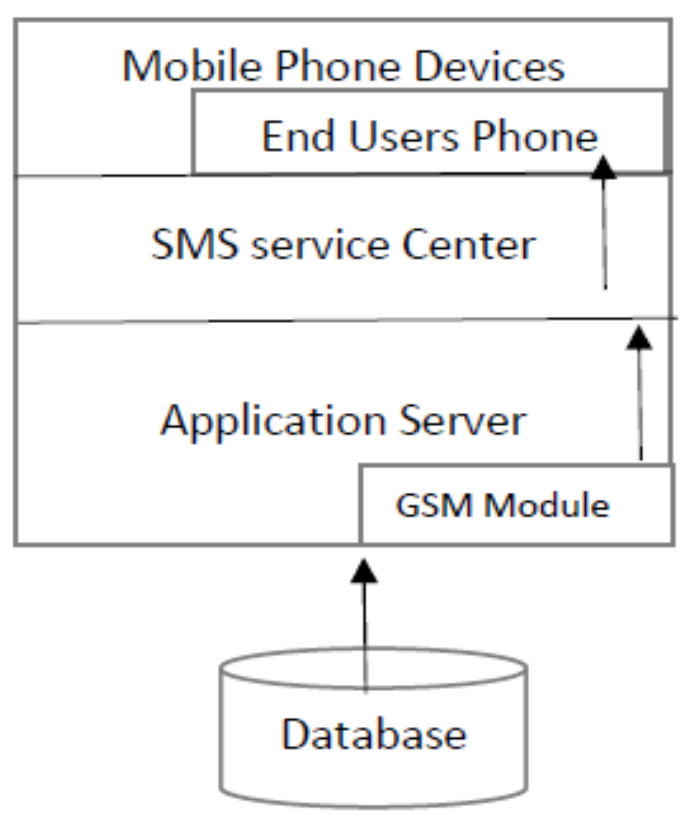

Fig 4: SMS System Architecture

A GSM module can be a devoted modem gadget or a standard GSM cell phone with a sequential, USB or Bluetooth affiliation and programming motive force to interface with a consecutive port or USB port of your pc or server as a monetarily smart reaction for sending SMS messages.

The form is to work with a database which contained the touch information of every body, for example, names and smartphone numbers, close by different information, as an example, the teach nuances, and lots of others they cope with, the begin time of each train, territory of the teach and variety of every assured.

\section{COMMON SHAPE}

Configuration Engineering manages the special UML [Unified Modeling language] documentations for the execution of task. The beneath format graph (Fig five) speaks to the inward planning unit getting equipped device, in which give up clients get suggested with the useful resource of the booking unit. whilst the train manner has been selected by means of manner of the teach dispatcher, it's miles refreshed to the planning unit and relying at the lighting fixtures sign device element the device went through the passage and transferred to the cloud. every enrolled end clients can get the replace from the cloud and plan their timetable as needs be. 


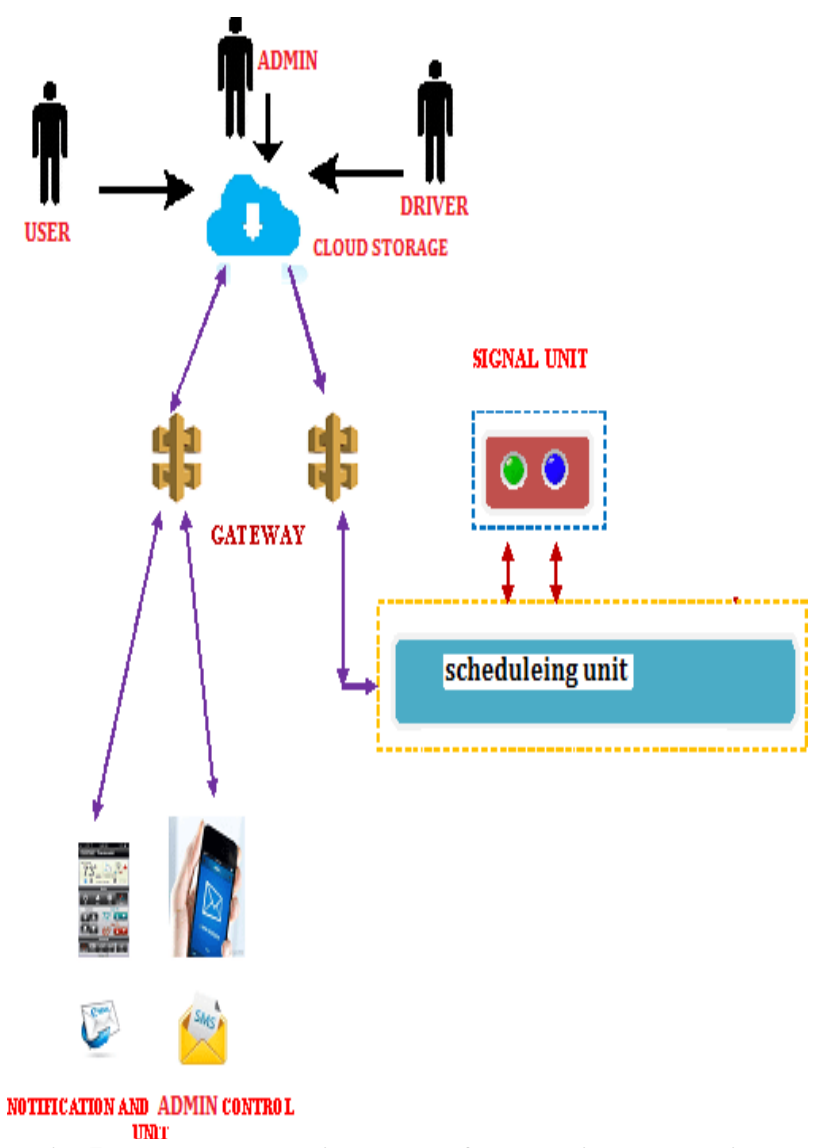

Fig 5: Internal architecture of the train scheduling.

\section{A.Use Case Diagram}

Use case graphs are considered for irregular state essential examination of a structure. Thusly, when the necessities of a structure are bankrupt down the functionalities are gotten being used cases. Toward the day's end, we can say that utilization cases are just the system functionalities and use cases are the on-screen characters who can speak with the structure. The on-screen characters can be human client or may be some inward or outside applications.

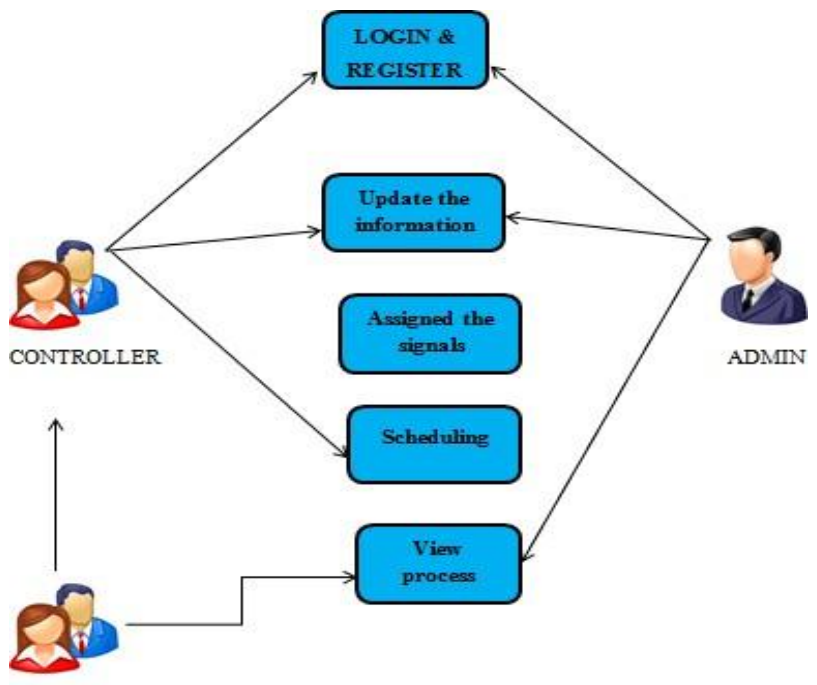

USER

Fig 6: Use case notation of the system design where the actors can update their respective task and make the scheduling process more efficient.

The above use case diagram (Fig 6) incorporates the fully-fledged system. each actor has their applicable task, wherein admin can get get entry to thru the all principal manner like Login \& Registration details, replace, and perspectives. If any changes that made unknowingly through the controller or person then admin could make this efficient and permits them to reschedule it again.

\section{B.Activity Diagram}

Activity is a particular operation of the system.

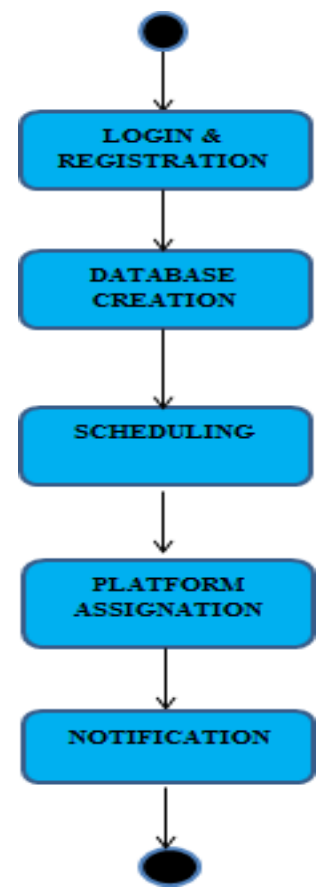

Fig 7: This activity life cycle circulates the whole process from the beginning to end of the scheduling unit.

motion isn't always used for imagining dynamic nature of a shape but they are furthermore used to accumulate the executable device with the aid of using forward and making revel in of techniques.

\section{V.RESULTS AND DISCUSSIONS}

Deliver us a threat to reflect onconsideration on a nonexistent condition with eight trains and five stations. four trains shifting located at A, transferring left to proper, at the same time as others are situated in E, transferring in inverse route because the discern (Fig eight) recommends. every station has 4 parallel tracks and there can be single track between stations. As appeared in under figure (Fig 9) each line demonstrates the improvement of a train. The even tendencies are stops, and vertical are developments of train. the extent dabbed strains display particular tracks internal station assets, and no sturdy strains are general to cowl inner these tracks. 


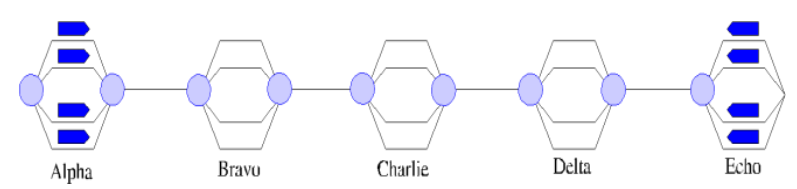

Fig 8: Initial State of the instance problem.

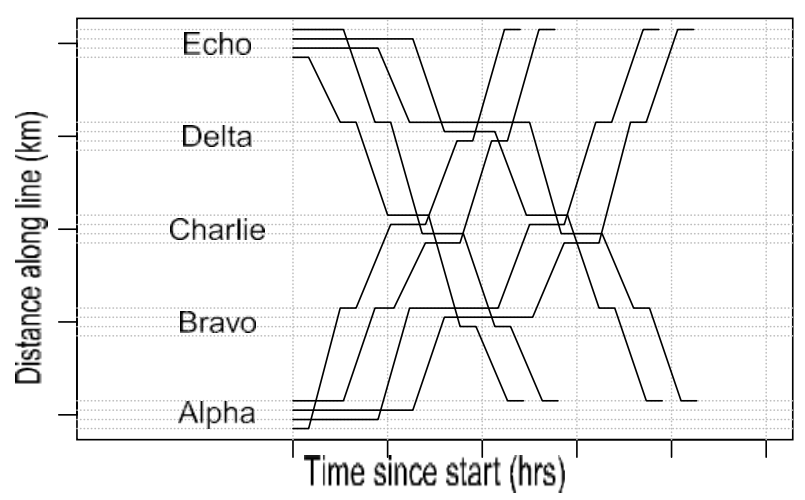

Fig 9: Test chart for 8 trains and 5 stations. Each solid line represents movement of one train.

[9] demonstrates the above investigation, and it is exhibited and examined in this flow explore. $\mathrm{P}$ is the quantity of states and HYP-1 contains trains with single need. $R\left(\_b+1+\_f\right)=19683$, with two activities for each state. Each state-activity pair is introduced with a triumph rate $\sigma(. \mathrm{x}, \mathrm{a})=$ half, prompting an underlying Q-Value of 0.5 or half. As the underneath figure (Fig 10) proposes The x-pivot demonstrates the Q-Value of the states, and the y-hub demonstrates the combined level of states that fall at or beneath the relating Q-Value. Toward the start, there is a stage change at 50 . The bend progresses toward becoming smoother with preparing. The distinction in the bends for 0 and 100 scenes is considerable for all Q-Values. The bends after 400 and 500 scenes are almost indistinguishable for low Q-Values, yet discernibly extraordinary for higher Q-Values.[9]

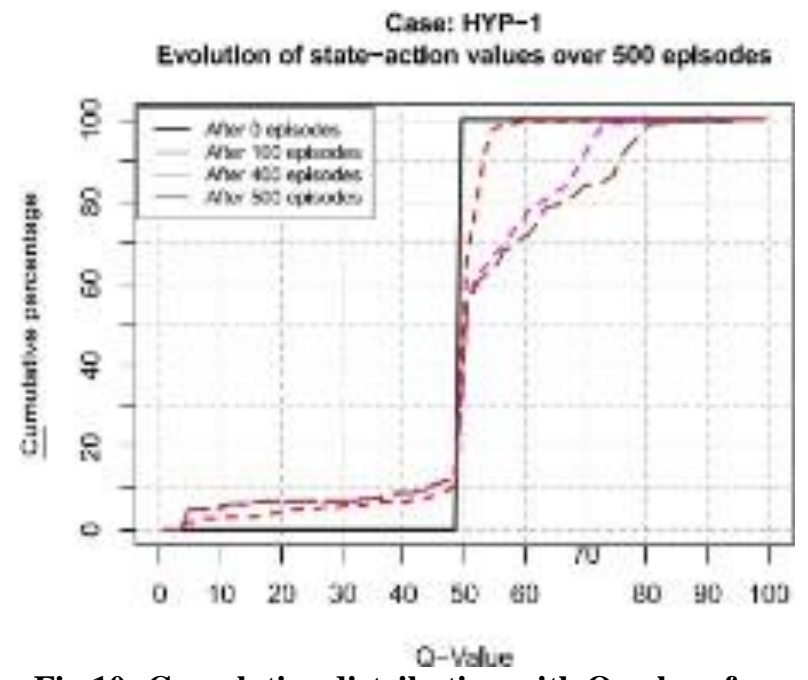

Fig 10: Cumulative distribution with $Q$ values for different test cases

\section{CONCLUSIONS}

This paper dissected a fortification getting to know method for arranging trains on railroad traces as examined in writing via [9]. The factor of convergence of the paper come to be on shape up a framework that could manipulate large, reasonable problem activities in estimation instances proportional with heuristic techniques, however with better timetable nice. The results given inside the past area exhibited that this intention modified into cultivated in at any charge 4 take a look at occasions, along with ones reliant on real timetables. The upsides of using RL as opposed to heuristics have been, (I) RL need to direct utilize the objective regards for managing the studying framework, (ii) it could change the Q-Values to abuse the houses of specific hassle events, (iii) it is able to entirety up starting with one difficulty case then onto the following, and (iv) it didn't require a exceptional deal of region bent for portraying the arranging policies.

\section{REFERENCES}

1. a brief Heuristic set of rules for the teach Unit venture problem with the resource of Valentina Cacchiani, Alberto Caprara_, and Paolo Toth. twelfth Workshop on Algorithmic methods for Transportation Modeling, Optimization, and structures (ATMOS'12).

2. perfect Scheduling of trains on a solitary line tune thru A. Higgins, E. Kozan and L. Ferreira. Res.- B. Vol. 30, No. 2, pp. 147-161, 1996

3. S. Liu and E. Kozan, "booking trains as a blocking off parallel-system career shop planning difficulty," Comput. Oper. Res., vol. 36, no. 10, pp. 2840-2852, 2009.

4. A Survey on trouble fashions and solution techniques to Rescheduling in Railway Networks by using Wei Fang, Member, IEEE, Shengxiang Yang, Senior Member, IEEE Xin Yao, Fellow, IEEE. Diary OF LATEX elegance documents, VOL.- , NO: - , JANUARY 2015

5. teach guiding principle in unmarried-song tour frameworks by way of using Marco Galaverna, Alberto Imovilli and Giuseppe Sciutto. vol 30,@ 1997 WIT Press. ISSN 1743-3509

6. R. Sutton and A. Barto, Reinforcement learning: An introduction, second ed. Cambridge, MA, u.s.: MIT Press, 2012.

7. S. Dündar and that i. Sahin, "teach re-making plans with hereditary calculations and artificial neural systems for unmarried-song railroads," Transp. Res. C, Emerg. Technol., vol. 27, pp. 1-15, Feb. 2013.

8. Yonan Getachew, "Plan and utilization of disaster and Emergency Reporting and reaction framework utilising SMS". extent 3, difficulty 5, can also 2014 ISSN 2277-8616.

9. Harshad Khadilkar,"A adaptable fortification learning set of rules for Scheduling Railway strains, IEEE Transactions on sensible Transportation systems, 2018. 